\title{
Retinex theory for color image enhancement: a systematic review
}

\author{
Ruaa Riyadh Hussein ${ }^{1}$, Yaser Issam Hamodi ${ }^{2}$, Rooa Adnan Sabri ${ }^{3}$ \\ ${ }^{1}$ College of Education for Girls, Al-Iraqia University, Iraq \\ ${ }^{2,3}$ Department of Computer Engineering, Ministry of Higher Education and Scientific Research, Iraq
}

\begin{tabular}{ll} 
Article Info & ABSTRACT \\
\cline { 3 - 3 } Article history: & $\begin{array}{l}\text { A short but comprehensive review of Retinex has been presented in this paper. } \\
\text { Retinex theory aims to explain human color perception. In addition, its } \\
\text { Received Jan 12, 2019 }\end{array}$ Revised Jul 19, 2019 \\
Accepted Jul 27, 2019 & $\begin{array}{l}\text { approaches for images contrast enhancement. In this review, the classical } \\
\text { theory of Retinex has been covered. Moreover, advance and improved } \\
\text { techniques of Retinex, proposed in the literature, have been addressed. }\end{array}$ \\
\hline Keywords: & $\begin{array}{l}\text { Strength and weakness aspects of each technique are discussed and compared. } \\
\text { An optimum parameter is needed to be determined to define the image } \\
\text { degradation level. Such parameter determination would help in quantifying } \\
\text { the amount of adjustment in the Retinex theory. Thus, a robust framework to } \\
\text { modify the reflectance component of the Retinex theory can be developed to } \\
\text { Contrast enhancement }\end{array}$ \\
Illumination & enhance the overall quality of color images. \\
Retinex &
\end{tabular}

Copyright (C) 2019 Institute of Advanced Engineering and Science. All rights reserved.

\section{Corresponding Author:}

Ruaa Riyadh Hussein,

College of Education for Girls,

Al-Iraqia University,

Baghdad, Iraq.

Email: abdul.ruaa@gmail.com

\section{INTRODUCTION}

The topic of image enhancement has drawn attention for being an essential approach to significantly improve images quality [1-5]. In this regard, the main purpose is to consider specific criteria to improve the quality of the image in a way that enhances the visual appearance of the resulting image when compared to the original image [6]. Add to this, the radiometric corrections to eliminate the effect of the radiometric errors that could exist even with the use of a calibrated imaging system to acquire the image. Examples are the non-uniform illumination, the impressive reduction transported by impressive sprinkling, and the preoccupation.

To date, a small number of methods based on single-scene image improvement is developed to provide a remedy for the issue of uneven and non-uniform illumination. The Homomorphic Filter method, denoted by (HF), can enhance the quality of the image by manipulation the illumination and enhance the dynamic compression of the image. Yet, the issue of color distortion is unavoidable. Furthermore, HF technique tends to bleach the image and its tonal rendition is poor [6-8]. On the other hand, the Histogram Equalization method, denoted by (HE), improves the intensity distribution of the image [6, 9-12]; however, the brightness and color of the original image are hardly preserved.

Color perception approaches are also proposed to solve the uneven intensity distribution issue. Here, the Human visual system (HVS) is utilized to apply the required illumination corrections in each and every pixel of the defect image. Figure1 shows a basic cross of the human eye. The retina of the human eye responds to very low levels of illumination through scotopic vision mode. Here, the rods of the retina become active to handle the low level of light brightness where the capability to distinguish colors vanishes. Other vision modes of human eyes are photopic where the cones are active and mesopic where the cones and 
rods are both active. Mesopic vision can be identified as a mixture of photonic and scotopic vision and happens under the circumstances when the level of illumination is low but not dark lighting situations as in most of the work offices $[13,14]$. Hence, at these circumstances of light levels, the pattern information contributed by the rods remains unclear. Thus the human visual observation (HVS) cannot recognize the color efficiently $[15,16]$. Yet, HVS is capable of observing the colors that are related to the sight i.e. color constancy. Hear, achieving invariance level in a certain illumination situation is feasible for many modern imaging applications. Examples include the contrast improvement in images that are dynamically created or the correction of white balance in the images that are produced by commercial cameras. Additional implementation model arises in the applications of remote sensing that involve image recognition.

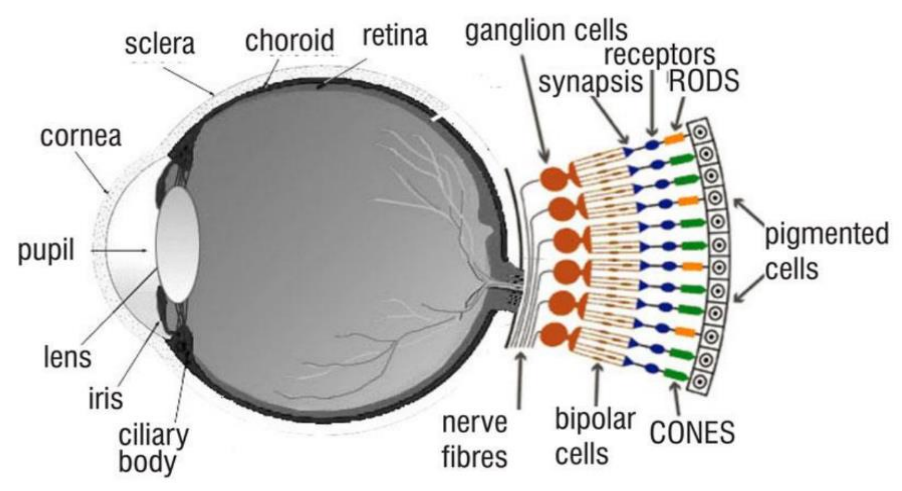

Figure 1. Basic cross of human eyes showing the rods and ones

Retinex Theory is fundamentally based on the visual perception property of human being. The theory is originally proposed by Land and McCann [6-8]. In their work, they tried to explain the HVS as a mixture of processes in both the cortex and the retina. The Retinex theory works in the illumination conditions that spatially vary with both of the color and the intensity. In addition, the insensitivity of the visual perception of the human being to the illumination that slowly changes is also observed by Retinex. Additional work by Edwin Land and McCann can be found in the study in [17].

Today, the Retinex theory has been applied in numerous different applications. Each and every one of those applications is particularly able to be adapted with specific tasks. Those tasks include the four main criteria which are: (1) contrast enhancement, (2) color balancing, (3) shadow removal in consumer electronics and imaging and (4) dynamic range compression. In this context, different assumptions are considered while applying the Retanex theory that in turn leads to various implementation results. Here, the assumptions of the theory are selected based on the considered applications as well. Furthermore, this theory, and in order for achieving images based on a reflectance correlation in the bandwidth of a wavelength, employs the function and the structure of the "retina and cortex" [6].

The reminder of this paper is arranged as follows: the theory of Retinex is presented in Section 2. In Section 3, Retinex theory types are further discussed where a thorough review has been made on each type. Strength and weakness of Retinex types are also highlighted. Section 4 presents the discussions and Section 5 concludes the review.

\section{THEORY OF RETINEX}

Retinex theory is first introduced by Land and Mc Cann as a model of "Human Visual Perception (HVS)" as mentioned-above [18]. In their work, the main consideration was based on the assumption that an acquired image is achieved through the combined action of the components of the reflectance and the illumination. Here, the illumination component captures all the types of light sources. Thus, the reflectance component can be isolated by excluding the illumination component from the acquired picture or image. Furthermore, the component of the reflectance handles the frequencies with high levels whereas the frequencies with low levels are captured by the illumination component.

A "Single-Scale Retinex" (SSR) [1] and a "Multi-Scale Retinex" (MSR) [19] were proposed by Jobson et al. Both of these methods apply a surround function on the input image. Consequently, the illumination level is estimated on the output image by utilizing the reflectance natural logarithm. However, a color distortion effect may be induced in the obtained image that represents a challenge in (SSR) and (MSR) 
methods. To fix this issue, a "Multi-Scale Retinex with Color Restoration" (MSRCR) was proposed [3]. Here, with (MSRCR), a step to handle color distortion and restoration using the color ratio of the red, green and blue (RGB) channels, is included. Yet, this technique tends to lose image details, especially in the bright region due to the globally applied mapping curve. To this end, the Retinex theory is basically based on the idea that the color perception is based significantly on the characteristics of the human vision neural system. Add to this, the term "Retinex" is formed by Land and McCann through the use of the two main root words "retina" and "cortex"; here, the vision process fundamentally relies on both the retina and cortex. Next, we refer to the following indication: when recognizing a color, the additional red color of a tungsten lamp is not identified by the eye. Hene, this indication was utilized by Edwin Land to introduce the Retinex method [20].

To this end, the Retinex approach, and since formulated by Land/ McCann, has become one of the popular approaches in image enhancement. The classical theory of Retinex has been modified and adaptively changed to meet specific criterias for various applications. The next section thoroughly explains the methods that had been derived from the original work of Land/ McCann.

\section{TYPES OF RETINEX}

Numerous Retinex approaches have been published over the years where they can be categorized into four main categories as follows, refer to Figure 2.

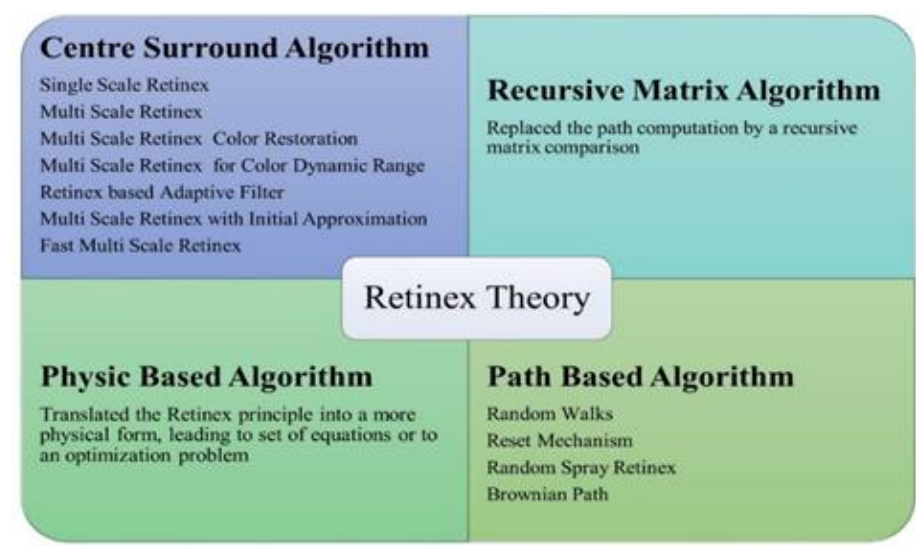

Figure 2. Types of retinex

\subsection{Path based algorithm}

The approach of the Path-Based Algorithm is to multiply the ratios along random walks to obtain the lightness of each pixel. The studies of Land and McCann [18, 21], are summarized here with the recent implementations such as those presented in $[22,23]$. The path based algorithm is divided into few categories as follows:

\subsubsection{Random walks}

The fundamental approach $\mathrm{f}$ this category is to examine the entire image by walking along all the 1-D paths. Accordingly, the relative relation between the light and shade is evaluated. Furthermore, the reflection values at each pixel are obtained; here, these values depend on the average light to shadow ratio of all the pixels of the image $[18,20]$.

Land and McCann concluded from several experiments that edges are the main features which are insusceptible to illumination and therefore be used as the basic material to attain color devotion [18]. Also comprehended the luminance ratio edge between two nearby points maintained the existing edges but the gentle slopes caused by the non-uniform illumination were eliminated. Therefore, the lightness values could be obtained by comprehensively exploring the luminance ratio in the image, see Figure 3 . Here, at each pixel where the lightnesses are evaluated, Retinex takes into account all the probable paths which have started and ended at random points and these pixels. In this context, this lightness along a path represents the average of the ratios between the intensity amounts of each succeeding edge points. Thus, a threshold is considered such that these ratios are set to be unitary if the difference between edge points is less, and hence, elimination the impact of the non-uniform illumination on the process. 


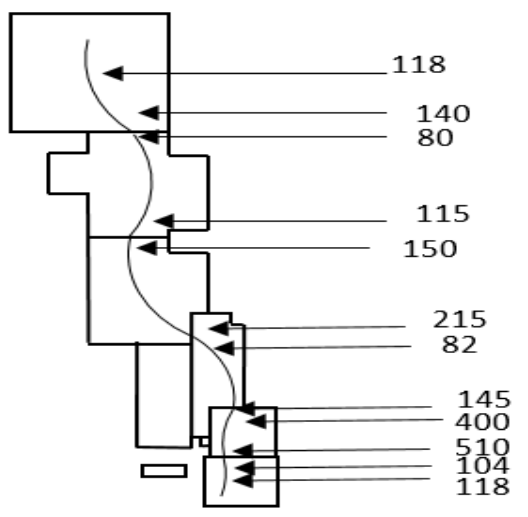

Figure 3. The random walk retinex mechanism by Land/McCann [6]

Then, to evaluate the lightness along all possible paths,

$$
L(x)=\frac{\sum_{k=1}^{N} L(x ; y k)}{N}
$$

Noted that L $\left(x ; y_{k}\right)$ would be evaluated as:

$$
L(x ; y k)=\sum_{t_{k}=1}^{n_{k}} \delta\left[\log \log \frac{I\left(x_{t_{k}}\right)}{I\left(x_{t_{k}}+1\right)}\right]
$$

Where $\gamma_{k}$ represents the considered path and $\mathrm{L}\left(x ; y_{k}\right)$ represents the relative lightness of a pixel $x$ with respect to a pixel $y_{k}$. Here, with a contrast threshold $(\mathrm{t})$ is considered

$$
\delta_{(s)}= \begin{cases}s, & \text { if }|s|>t \\ 0, & \text { if }|s|<t\end{cases}
$$

\subsubsection{Reset mechanism}

The aforementioned definition was improved in the reset mechanism. This approach ensures to consider the regions that include the highest values of luminance such that all the paths emanating from these regions are counted. However, areas that consist of the highest reflectance are not easily distinguishable. The development of this technique is droved based on the assumption that the initial ratio is given. Then, the signal ratio is calculated between the first and the second receptors and subsequent by the ratio between the second and the third receptors, and so on (4) states the expression by which the rest mechanism of Retinex is formulated.

$$
\forall j=1, \ldots, n_{k}-1, \sum_{t_{k}=j}^{n_{k}} \delta\left[\log \frac{I\left(x_{t_{k}}\right)}{\left(x_{t_{k}}+1\right)}\right] \leq 0
$$

Here, the average is evaluated along the paths such that the overall summation consists of non-positive partial summations as shown in (4).

\subsection{Recursive matrix algorithm}

The Recursive Matrix Algorithm was initiated by Frankle and McCann [24]. Here, a recursive matrix comparison approach is utilized as an alternative to the original path computation [24-26]. This algorithm crucially depends on the calculation of the optimum number of iterations; that is the number of visits to a pixel's neighbors. Hence, it is considered as a limitation since this parameter is hard to be determined and can essentially affect the final result [27]. Figure 4 show implementation of Brownian Path Algorithm. 


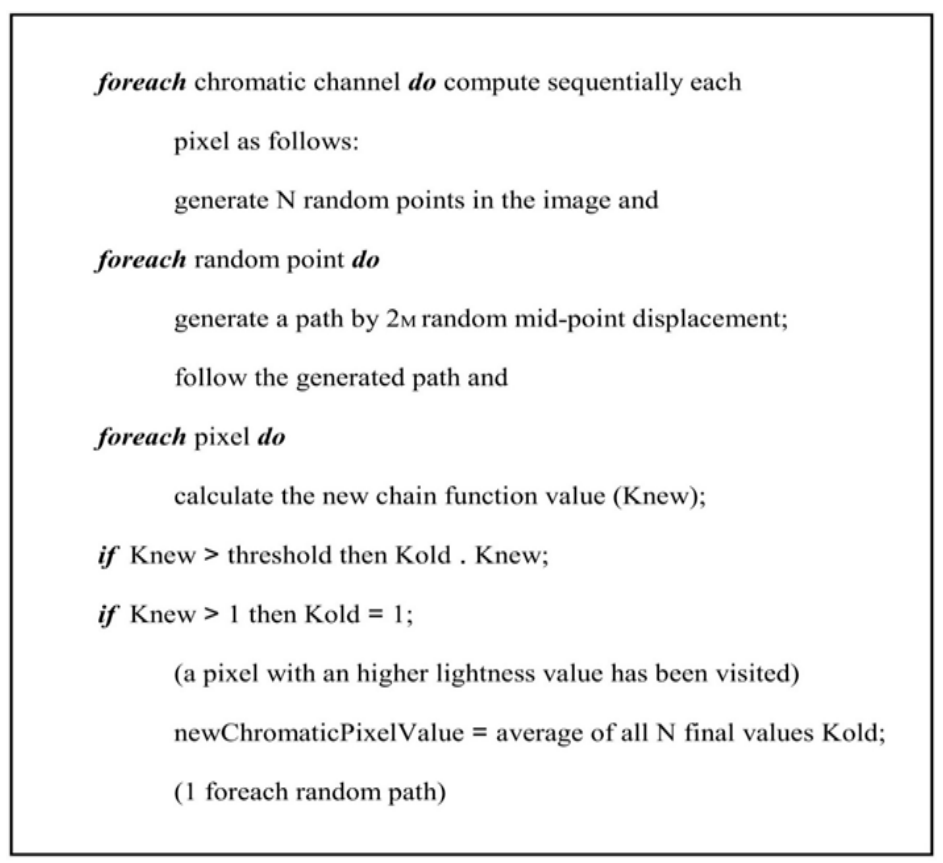

Figure 4. Implementation of brownian path algorithm

\subsection{Physics-based algorithm}

This algorithm represents one of the main Retinex division with a physical form that is presented by a set of equations [28-30]. In this algorithm, image intensity is denoted by I, the reflectance is denoted by $\mathrm{R}$, while L refers to the incident illumination intensity. Consequently, the equation is presented as:

$$
I=R \cdot L
$$

In this regard, a different approach to evaluate the illumination, as well as the reflectance, was proposed by Horn. In his approach, Horn utilizes the logarithm of the image intensity such that,

$$
\log I=\log R+\log L
$$

The main concept of Retinex Physics-based algorithm is that illumination might contain varies smoothly over the image. On the other hand, sharp discontinuities are shown only during reflectance changes. Laplacian function stated in (7) was also utilized by Horn to introduce "Poisson equation".

$$
\Delta \log I=\Delta \log R+\Delta \log L
$$

Accordingly, Horn proposed another technique that applies the threshold operator $T$ in-order to remove the second term of (7) that is constant, and thus provided a direct relation between $R$ and $I$ as shown in the (8).

$$
\Delta(\log R)=T(\Delta \log I)
$$

The "Poisson equation" successfully estimates the value of reflectance. The result is normalized by assuming one point in the image as minimum that has the ability to reflect all the lights that assumed to be white. This equation is also denoted by "Poisson-equation-type Retinex algorithm" in [31]. The study by Kimmel et al. [32] also proposed the variation models for Retinex. In this context, the approach considers the illumination to be smooths. Thus, it inserts the variation model in a similar manner to the Horn's model. Active multiresolution solution to various problems are also introduced by the authors. 


\section{DISCUSSIONS}

\subsection{Summary of the research}

The main objective of this study is to identify the potential of Retinex algorithm in image enhancement applications. A systematic search of the literature revealed the classical approaches of Retinex algorithm such as in Path-Based and Recursive Matrix Algorithms. These algorithms are among the first findings by Land and Mc-Cann where the implementation of both techniques are based on the path computation. The more advanced approach has been implemented in the latter algorithm where the path used in the first algorithm was replaced with the recursive matrix comparison. Retinex techniques are simplified in Figure 5.

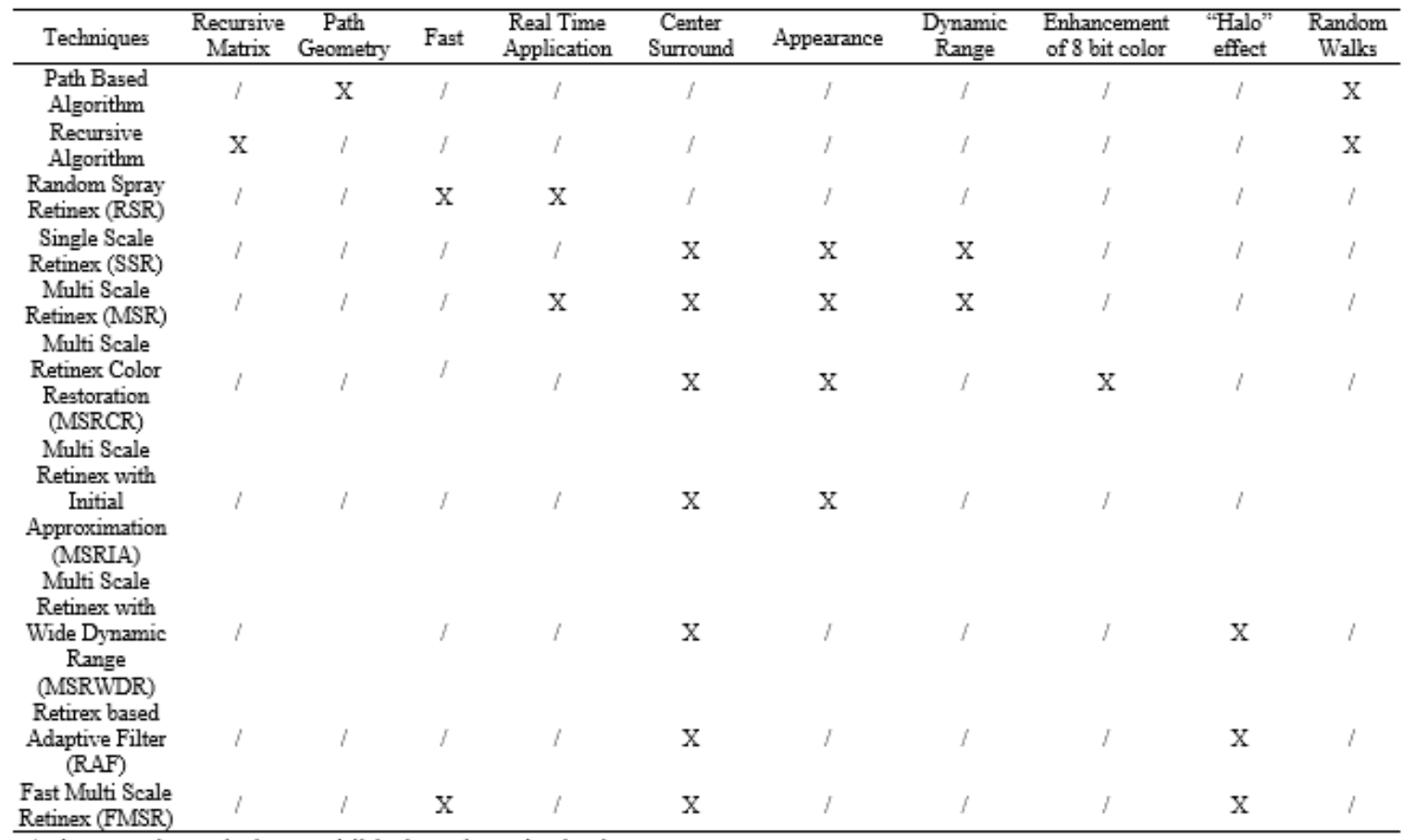

/ - denotes the technique exhibit the selected criteria

$\mathrm{X}$ - denotes the technique does not exhibit the selected criteria

Figure 5. Retinex methods techniques

The complexity of generating a path that includes a number of paths to be used, and their length are mainly solved in the Recursive Matrix Algorithm. However, the final results of the algorithm highly depend on iteration that indicates the time pixel boundaries in the image and determine the number of the neighbors to be considered. Hence, the objective of image enhancement is subjected to this crucial parameter which adds more complexity to the enhancement task itself.

The Retinex theory has been further improved by including the neighborhood or surround function of each pixel. The limitations in SSR such as color rendition and dynamic range compressions were improved by the MSR function. To date, numerous works of literature have reported on the modifications of the Retinex theory based on this MSR function as in [33-35]. The trade-off between preserving color distribution and suppressing halo effect on the image is still unresolved. The weighted function needs to be designed such that the halo effect will be eliminated while the nature color of the image will not be distorted. In addition, noises of the image should not be enlarged.

The optimization factor was introduced in the Physic Based Algorithm where the new factor plays a major role in obtaining the enhanced image without compromising the natural color of the image. The improvements that has made to the Retinex theory has led to the exploration of the new possibility in enhancing the image without introducing halo effect while maintaining color distribution of the image. 


\subsection{Applications of retinex}

\subsubsection{Color balancing/restoration}

Color balancing and color restoration have been presented and described through different ways and models by many researchers [1, 2, 36-41]. Retinex theory is always there to help for achieving these objectives. In [42] a new spectrum shape elements approach to handle the non-isopalmitic deviation in illumination was presented. This approach was implemented and tested for floor and hyperspectral images. While in [43], MSR method had been used to examine the visual system of human being and illustrates how it perceives color. A slightly different approach of MSR is reported in [44]. In this regard, the characteristics of the nighttime image are utilized to modify Retinex. The tradition algorithm was replaced by a customized sigmoid function using an improved framework. In summary, the Retinex theory maintains the values of the RGB color components of each pixel of the image and estimates the reflectance of each point.

\subsubsection{Shadow removal}

Retinex theory has been known as one of the solutions in shadow removal. In this regard, the SSR algorithm is utilized in the study in [45] where the shadow of an image with a single color was eliminated. Firstly, shadow and non-shadow regions are evaluated by SSR. Here, and by considering the features of SSR, a robust dynamic compression is achieved using a limited scale filter and thus the shadow is removed. While in [46] MSRCR was utilized for shadow removal. Here, the shadow area is observed using a detection approach that is based on a gradient edge which is combined with 1-D illumination invariant image. Subsequently, the Retinex algorithm is used to remove the shadow where the brightness of the shadow area will be adjusted as the final step. In the study by [47], MSR method was applied to enhance the shadow regions for an illuminant independent skin color detection. In addition, MSR was utilized in [48] to detect the vehicle shadow and then eliminate it.

\subsubsection{Biomedical applications}

Retinex has taken a big part of being used in medical imaging applications [49-53]. In [51] Retinex was applied to be used in automatic analysis correctness of skin lesions. In this regard, MSR was used to enhance the image of the spinal cord for medical purposes. Here, the core of this algorithm is the design of Gaussian surround function. In this regard, image improvement in medical applications mostly consider the spinal cord images. This is because enhancing the details of the spinal cord images lead to an increase in the accuracy of the diagnosis. On the other hand, the author of the study in [52] introduced a real-time image enhancement for gastric endoscopy, by applying the Retinex image enhancement technique on video images. MSRCR had also been employed in [53] to reduce the contrast between arteries and vein increase classification of blood vessels in retinal images. In addition to the three main applications discussed above, Table 1 presents the optimization parameters and applications of Retinex in various fields.

Table 1. Optimization parameters and applications for retinex techniques

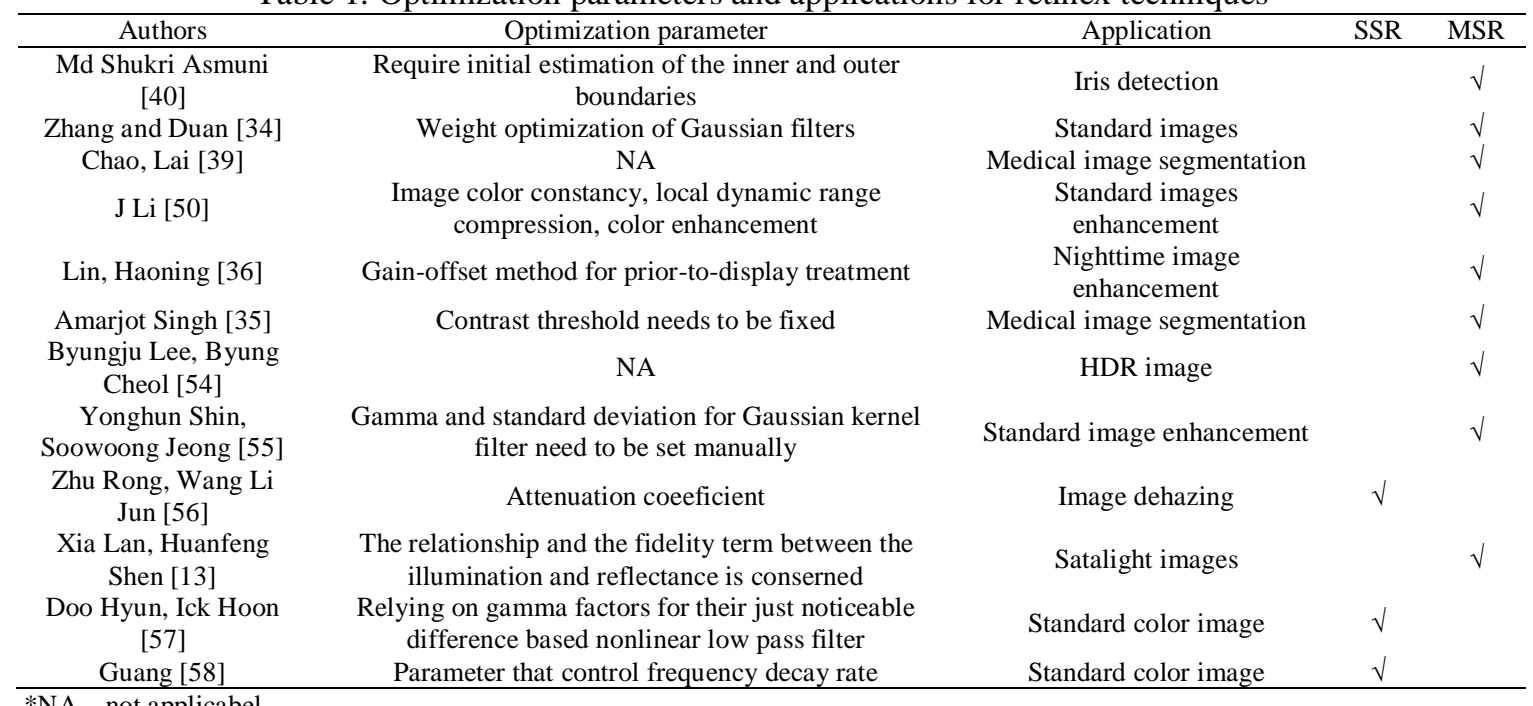




\subsection{Recommendation for future research}

Based on the studies included in this review, some recommendations for future studies of Retnix can be proposed. Firstly, as the review summarized in the previous section, it can be observed although improvements have been made to the original Retinex theory, most algorithms are relying on the optimization parameters. The parameters need to be manually set where the performance of the improved algorithms are highly affected. The selection of the optimum parameter could be biased and not consistent to be used as a standard framework for Retinex enhancement. Therefore, a model to reduce the dependency on the optimization parameter could be developed.

Improvements can also be made on the existing techniques where quantifiable parameter to reflect on the degree of the image degradation can be further investigated. To date, there is no single measurement could represent the degradation level of the image. Here, human conception is mainly utilized to address the aspect of images quality. Hence, future studies could be implemented to explore the degradation level of the image where the level of contrast, illumination, and image details are considered. In this regard, a formulation of the optimum parameter that represents these factors could be proposed in order to define the level of image degradation.

Addition improvements on the existing Retinex theory could be investigated to enhance the overall illumination of the image. Here, the dynamic range compression in the reflectance components can be further pushed. Finally, a unifying framework to modify the reflectance components of Retinex theory will be also beneficial for image enhancement to be applied in various applications, see Table 1.

\section{CONCLUSIONS}

This paper presents the variation of the Retinex algorithm models for uneven intensity correction. Here, strength and weakness aspects of every implementation theory are highlighted and discussed. Furthermore, the relationship between the illumination and reflectance is addressed. In summary, this paper illustrates the applications and approaches the implementation of the Retinex theory. In addition, a robust framework of modifying the reflectance components of the Retinex could be explored and further improved.

\section{REFERENCES}

[1] D. J. Jobson, et al., "Properties and performance of a center/surround retinex," Image Processing, IEEE Transactions on, vol. 6, pp. 451-462, 1997.

[2] D. J. Jobson, et al., "A multiscale retinex for bridging the gap between color images and the human observation of scenes," Image Processing, IEEE Transactions on, vol. 6, pp. 965-976, 1997.

[3] Z. U. Rahman, et al., "Retinex processing for automatic image enhancement," Journal of Electronic Imaging, vol. 13, pp. 100-110, 2004.

[4] C. Shaohua and A. Beghdadi, "Natural Rendering of Color Image based on Retinex," Image Processing (ICIP), 2009 16th IEEE International Conference on, 2009.

[5] W. Shuhang, et al., "Naturalness Preserved Enhancement Algorithm for Non-Uniform Illumination Images," Image Processing, IEEE Transactions on, vol. 22, pp. 3538-3548, 2013.

[6] A. K. Vishwakarma and A. Mishra, "Color image enhancement techniques: a critical review," Indian J. Comput. Sci. Eng, vol. 3, pp. 39-45, 2012.

[7] M. F. Zakaria, et al., "A review: Image compensation techniques," Computer Engineering and Technology (ICCET), 2010 2nd International Conference on, 2010.

[8] M. D. Dileep and A. S. Murthy, "A comparison between different colour image contrast enhancement algorithms," Emerging Trends in Electrical and Computer Technology (ICETECT), 2011 International Conference on, 2011.

[9] N. Sengee and H. Choi, "Brightness preserving weight clustering histogram equalization," Consumer Electronics, IEEE Transactions on, vol. 54, pp. 1329-1337, 2008.

[10] H. Yeganeh, et al., "A novel approach for contrast enhancement based on histogram equalization," Computer and Communication Engineering, 2008. ICCCE 2008. International Conference on, 2008.

[11] G. Thomas, et al., "Histogram specification: a fast and flexible method to process digital images," Instrumentation and Measurement, IEEE Transactions on, vol. 60, pp. 1565-1578, 2011.

[12] N. Sengee, et al., "Image contrast enhancement using bi-histogram equalization with neighborhood metrics," Consumer Electronics, IEEE Transactions on, vol. 56, pp. 2727-2734, 2010.

[13] X. Lan, et al., "A spatially adaptive retinex variational model for the uneven intensity correction of remote sensing images," Signal Processing, vol. 101, pp. 19-34, 2014.

[14] A. Stockman and L. T. Sharpe, "Into the twilight zone: the complexities of mesopic vision and luminous efficiency," Ophthalmic and Physiological Optics, vol. 26, pp. 225-239, 2006.

[15] M. Song, et al., "Probabilistic exposure fusion," Image Processing, IEEE Transactions on, vol. 21, pp. 341-357, 2012.

[16] M. Song, et al., "Color to gray: visual cue preservation," Pattern Analysis and Machine Intelligence, IEEE Transactions on, vol. 32, pp. 1537-1552, 2010. 
[17] J. J. McCann, et al., "Quantitative studies in retinex theory a comparison between theoretical predictions and observer responses to the "color mondrian" experiments," Vision Research, vol. 16, pp. 445-IN3, 1976.

[18] E. H. Land and J. McCann, "Lightness and retinex theory," JOSA, vol. 61, pp. 1-11, 1971.

[19] D. J. Jobson, et al., "A multiscale retinex for bridging the gap between color images and the human observation of scenes," Image Processing, IEEE Transactions on, vol. 6, pp. 965-976, 1997.

[20] E. H. Land, "The retinex theory of color vision," Scientific America, 1977.

[21] E. H. Land, "Recent advances in retinex theory," Vision research, vol. 26, pp. 7-21, 1986.

[22] D. Marini and A. Rizzi, "A computational approach to color adaptation effects," Image and Vision Computing, vol. 18, pp. 1005-1014, 2000.

[23] E. Provenzi, et al., "Mathematical definition and analysis of the Retinex algorithm," Journal of the Optical Society of America A, vol. 22, pp. 2613-2621, 2005.

[24] J. A. Frankle and J. J. McCann, "Method and apparatus for lightness imaging," Google Patents, 1983.

[25] J. McCann, "Lessons learned from Mondrians applied to real images and color gamuts," Color and Imaging Conference, Society for Imaging Science and Technology, 1999.

[26] B. Funt, et al., "Retinex in MATLAB" ," Journal of electronic imaging, vol. 13, pp. 48-57, 2004.

[27] F. Ciurea and B. Funt, "Tuning retinex parameters," Journal of Electronic Imaging, vol. 13, pp. 58-64, 2004.

[28] A. Blake, "Boundary conditions for lightness computation in Mondrian world," Computer Vision, Graphics, and Image Processing, vol. 32, pp. 314-327, 1985.

[29] B. V. Funt, et al., "Recovering shading from color images," Computer Vision-ECCV'92, Springer, 1992.

[30] D. Terzopoulos, "Image analysis using multigrid relaxation methods," Pattern Analysis and Machine Intelligence, IEEE Transactions on, vol. 2, pp. 129-139, 1986.

[31] H. Takahashi, et al., "Variational Retinex Algorithm with its Application to a High - Quality Chroma Key," Image Processing, 2006 IEEE International Conference on, 2006.

[32] R. Kimmel, et al., "A variational framework for retinex," International Journal of computer vision, vol. 52, pp. 7-23, 2003.

[33] Y. Jindong, et al., "Research on submicron measurement based on computer micro-vision," Manipulation, Manufacturing and Measurement on the Nanoscale (3M-NANO), 2013 International Conference on, 2013.

[34] Q. Zhang and H. Duan, "Biological weight selection of multi-scale retinex via artificial bee colony algorithm," Optik - International Journal for Light and Electron Optics, vol. 125, pp. 1434-1438, 2014.

[35] J. Li, "Application of image enhancement method for digital images based on Retinex theory," Optik - International Journal for Light and Electron Optics, vol. 124, pp. 5986-5988, 2013.

[36] V. Vonikakis, et al., "Fast centre-surround contrast modification," Image Processing, IET, vol. 2, pp. 19-34, 2008.

[37] W. Hao, et al., "Retinex-Like Method for Image Enhancement in Poor Visibility Conditions," Procedia Engineering, vol. 15, pp. 2798-2803, 2011.

[38] L. Tang, et al., "Improved Retinex Image Enhancement Algorithm," Procedia Environmental Sciences, vol. 11, pp. 208-212, 2011.

[39] W. H. Chao, et al., "Correction of inhomogeneous magnetic resonance images using multiscale retinex for segmentation accuracy improvement," Biomedical Signal Processing and Control, vol. 7, pp. 129-140, 2012.

[40] D. y. S. Md Shukri, et al., "An improved multiscale retinex algorithm for motion-blurred iris images to minimize the intra-individual variations," Pattern Recognition Letters, vol. 34, pp. 1071-1077, 2013.

[41] H. K. Cheung, et al., "An efficient retinex-like brightness normalization method for coding camera flashes and strong brightness variation in videos," Signal Processing: Image Communication, vol. 25, pp. 143-162, 2010.

[42] A. Nikonorov, et al., "Spectrum shape elements model to correct color and hyperspectral images," Pattern Recognition in Remote Sensing (PRRS), 2014 8th IAPR Workshop on, 2014.

[43] W. Liqian, et al., "Variational Bayesian Method for Retinex," Image Processing, IEEE Transactions on, vol. 23, pp. 3381-3396, 2014.

[44] H. Lin and Z. Shi, "Multi-scale retinex improvement for nighttime image enhancement," Optik - International Journal for Light and Electron Optics, vol. 125, pp. 7143-7148, 2014.

[45] Y. Kang and T. Deshou, "Shadow Removal from Images Using an Improved Single-Scale Retinex Color Restoration Algorithm," Computational Sciences and Optimization, 2009. CSO 2009. International Joint Conference on, 2009.

[46] M. Guowen and Y. Jinfeng, "Shadow removal using Retinex theory," Intelligent Visual Surveillance (IVS), 2011 Third Chinese Conference on, 2011.

[47] T. Yongqiu, et al., "Skin color detection by illumination estimation and normalization in shadow regions," Information and Automation (ICIA), 2010 IEEE International Conference on, 2010.

[48] W. Qi-sheng, et al., "An Improved Multi-Scale Retinex Algorithm for Vehicle Shadow Elimination Based on Variational Kimmel," Ubiquitous Intelligence \& Computing and 7th International Conference on Autonomic \& Trusted Computing (UIC/ATC), 2010 7th International Conference on, 2010.

[49] Q. Meng, et al., "Improved Multi-Scale Retinex Algorithm for Medical Image Enhancement," Information Engineering and Applications, R. Zhu and Y. Ma, Editors, Springer London, pp. 930-937, 2012.

[50] S. Setty, et al., "Development of multiscale retinex algorithm for medical image enhancement based on multi-rate sampling," Signal Processing Image Processing \& Pattern Recognition (ICSIPR), 2013 International Conference on, 2013.

[51] L. Yanan, et al., "No Reference Uneven Illumination Assessment for Dermoscopy Images," Signal Processing Letters, IEEE, vol. 22, pp. 534-538, 2015. 
[52] H. Okuhata, et al., "Application of the real-time Retinex image enhancement for endoscopic images," Engineering in Medicine and Biology Society (EMBC), 2013 35th Annual International Conference of the IEEE, 2013.

[53] Q. Mirsharif, et al., "Automated characterization of blood vessels as arteries and veins in retinal images," Computerized Medical Imaging and Graphics, vol. 37, pp. 607-617, 2013.

[54] Lee, B. and B. Cheol Song, Multi-image high dynamic range algorithm using a hybrid camera. Signal Processing: Image Communication, 30(0): p. 37-56, 2015.

[55] Yonghun, S., J. Soowoong, and L. Sangkeun. Content awareness-based color image enhancement. in Consumer Electronics (ISCE 2014), The 18th IEEE International Symposium on. 2014.

[56] Rong, Z. and W.L. Jun, Improved wavelet transform algorithm for single image dehazing. Optik - International Journal for Light and Electron Optics, 125(13): p. 3064-3066, 2014.

[57] Doo Hyun, C., et al. Color image enhancement using single-scale retinex based on an improved image formation model. in Signal Processing Conference, 2008 16th European. 2008.

[58] Guang, Q., The Research on a New Image Enhancement Algorithm Based on Retinex Theory, in Advanced Research on Computer Education, Simulation and Modeling, S. Lin and X. Huang, Editors, Springer Berlin Heidelberg, p. 336-342, 2011.

\section{BIOGRAPHIES OF AUTHORS}

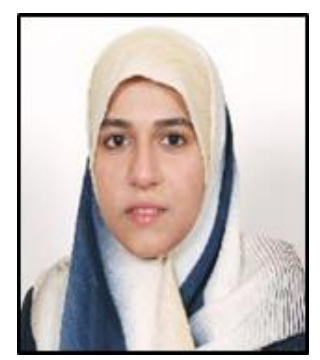

Ruaa Riyadh Hussein received B.Sc. degree in Computer Science- College Of Education- Ibn alHaytham, Baghdad University, Baghdad, Iraq, in 2007, and M.Sc degree in Computer ScienceCollege of Engineering and Computer Science, University of Central Florida, Florida, USA, in 2014. She is curentlly a teacher in College of Education for Girls, Al-Iraqia University, Iraq.

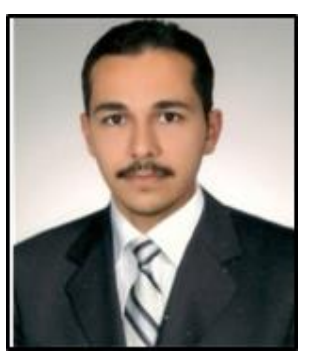

Yaser Issam Hamodi received B.Sc., degrees in Computer Engineering - College of Electrical and Electronic Techniques, Foundation of Technical Education, Baghdad, Iraq, in 2004, and M.Sc degree in computer engineering from Cankaya University - Ankara-Turkey in 2012.

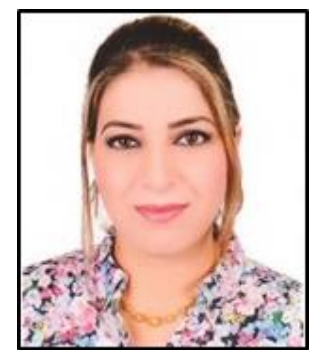

Rooa Adnan Sabri received B.Sc. degree in Computer Engineering- College of Engineering, Al-Mustansiriya University, Baghdad, Iraq, in 2003, and M.Sc degree in Computer EngineeringDepartment of Computer Engineering, University of Technology, Baghdad, Iraq, in 2014. 